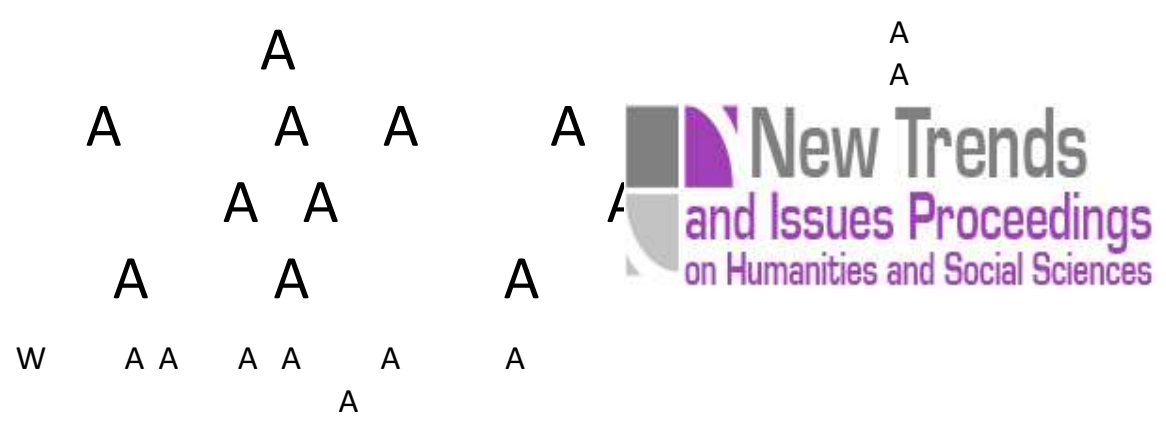

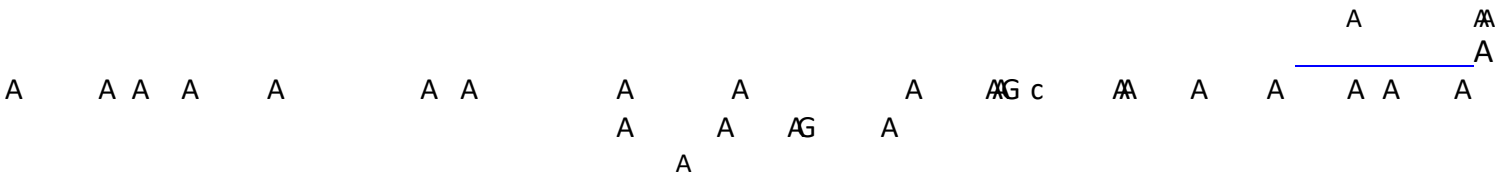

The learning activities of green university for environmental education undergraduate students

Prayoon Wongchantra ${ }^{\mathrm{a}^{*}} \mathrm{~A}$

$\mathrm{N}$

$$
\mathrm{N}
$$

Khuantean Wongchantra ${ }^{b} \mathrm{~A}$ AN

Likhit Junkaew ${ }^{\mathrm{C}} \mathrm{A}$

$\mathrm{N}$

Kannika Sukngam ${ }^{d} A$

$\mathrm{N}$

Suparat Ongon ${ }^{\mathrm{e}} \mathrm{A}$ $\mathrm{N}$

Ubon Kwaenthaisong ${ }^{f} A$

$\mathrm{N}$

A

Suggested Citation:
A $A$
A A
A A
A A A A A

A A

C

AG

A A

A A

AG $A$ A

A A $\quad A$

A A

A

A $A$ A

A A $A$

A $\quad A$

A

A A A

A $\quad A$

A

A $A$

A $\mathrm{A}$

AG A $A$

A

AG

A A

A

AG A A

A

AG $\quad A \quad A$

A A A

Proceedings on Humanities and Social Sciences.

A
A A A
A A
C $A$ A

A $A A$
$\begin{array}{llllll}A & A & A & A & A\end{array}$ A

Abstract

\begin{tabular}{|c|c|c|c|c|c|c|c|c|c|c|c|c|c|c|c|c|c|c|c|c|c|c|c|c|}
\hline A & $A A$ & $A$ & & $A$ & A A & A & A & A & A & & A & A & & $A$ & A A & A & A & $A$ & & A A & A & & & A \\
\hline A & A & A & A & & & A & a & A & A & & A & & A & & AG & A & A & & A & A & 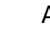 & A & & \\
\hline$V$ & & & & A & & $A A$ & A & $A \quad A$ & & A & A & & A & A & & A & A & A & A & A & & & A & A \\
\hline A & A $A$ & & A & & $A A$ & & & $A$ & A & A & & A & $A$ & & A & A & A $A$ & & A & $A$ & $A C$ & & A & A \\
\hline$A$ & & A & A & & $A$ & $A$ & A & $A A$ & & $A C$ & A & A & & A & A & A & A A & & A & A & A & & A & \\
\hline$A$ & A A & & A & $A$ & & $A$ & $A$ & & A & A & $A$ & $A$ & A & A & $A A$ & & A $A$ & 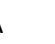 & A & $A$ & & $A$ & & \\
\hline$A$ & A & A & A & & A & A $A$ & & & A A & A & A & A & & A & A & A A & A & A & & A A & & $A$ & A & \\
\hline A & $\mathrm{A} A$ & & & A & A & & A & A & A & A & & A & A & $x$ & A & $A$ & A & & & & & & & \\
\hline
\end{tabular}
A $\begin{array}{llllllll}\text { A } & \text { A } & \text { A } & \text { A } & \text { A } & \text { A } & \text { A }\end{array}$

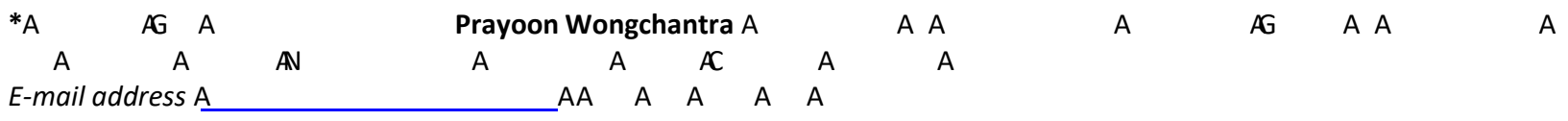




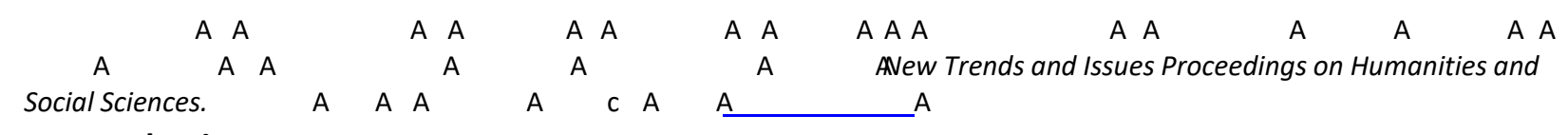

1. Introduction

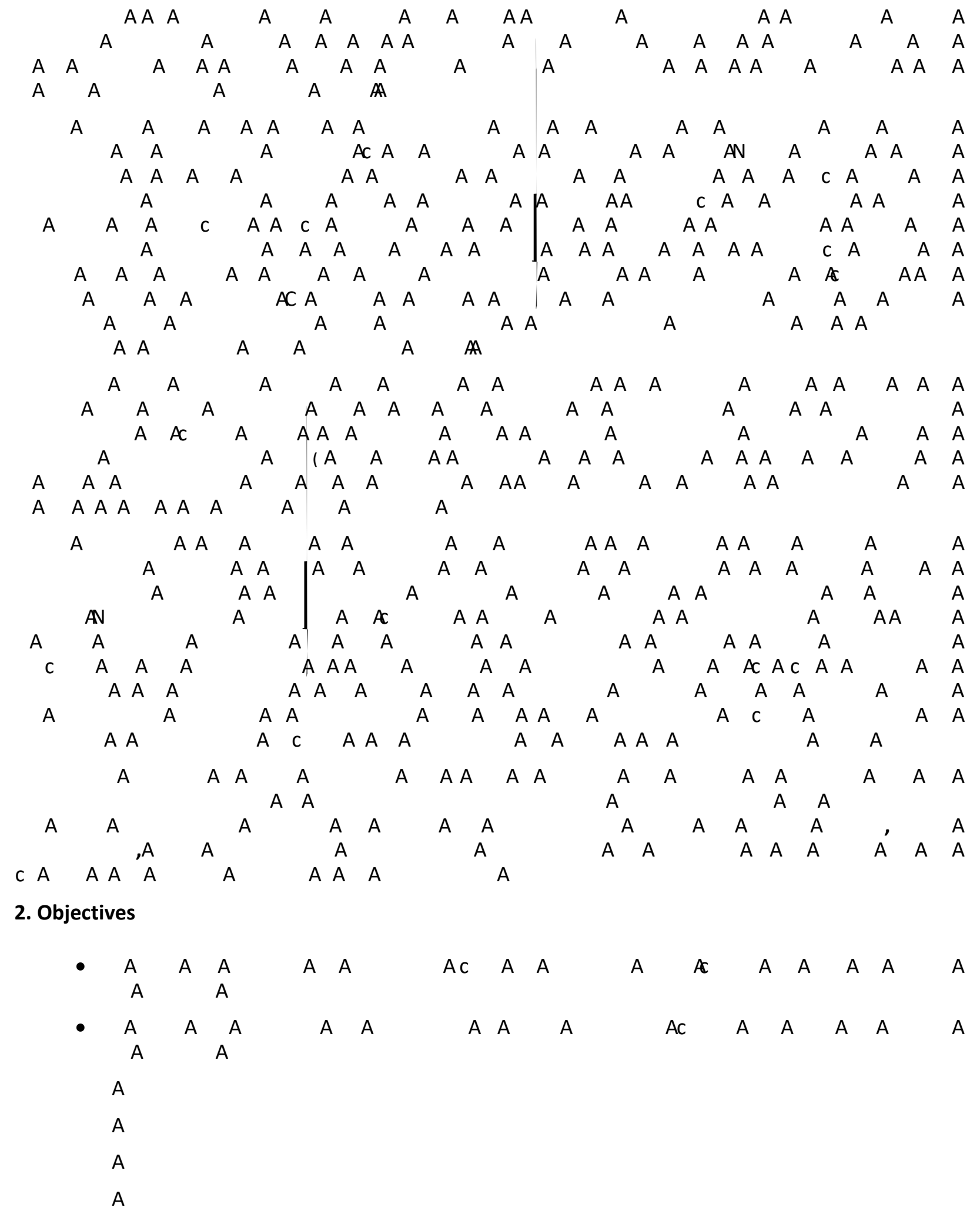




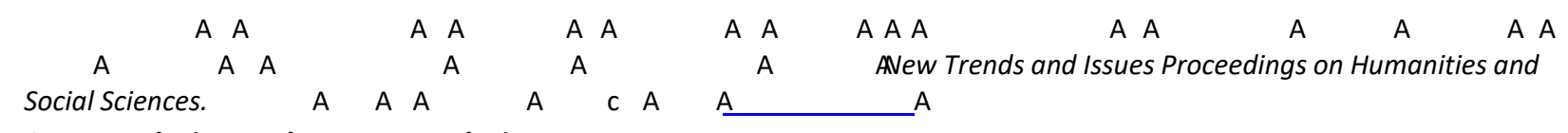

3.1. Population and target population

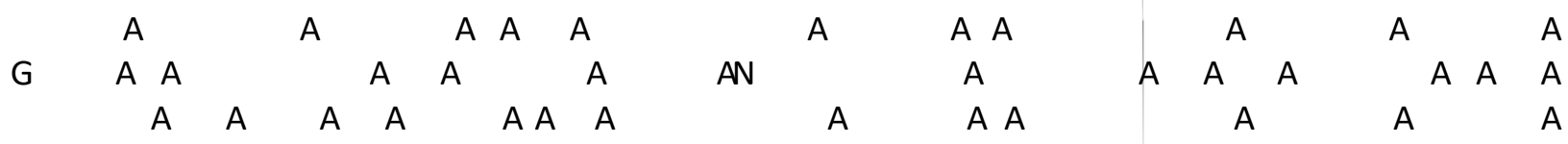
G A A
A $A$ A $C$ C A A
A $A$

$\mathrm{N}$

A

\subsection{Tool used in the research}

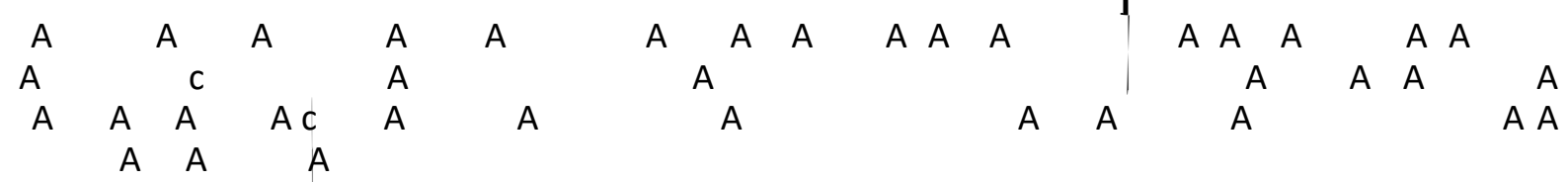

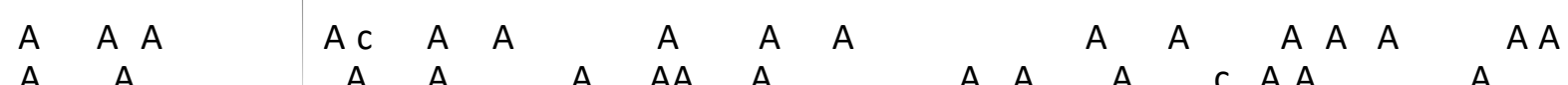

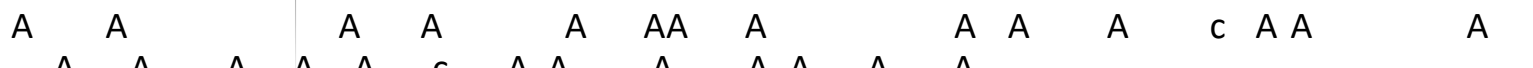
$A \quad A \quad A \quad A \quad A \quad C \quad A A$ A $A A$ A $A$

$\left.N \quad A \quad A\right|^{A} A$ A A $A$ A $A$ A A A A A A A A A A A $A$

4. Research Steps
A
\begin{tabular}{lll|l}
$A$ & $A$ & $A$ & $A$ \\
$A$ & $A$ & $A$
\end{tabular}
A Ac A A
A $\quad A$
A $A \quad A$
A $\quad A \quad A \quad A$
A A A A A A $A A$
A $A$ A
A A C A
A $\quad A \quad A$

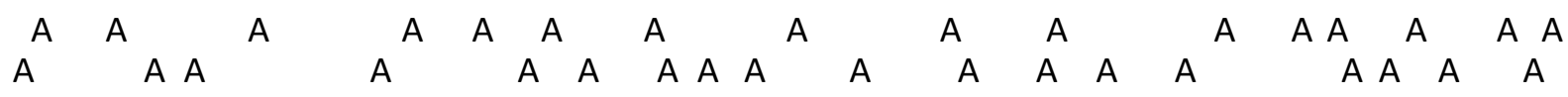 A $\quad$ A $\quad$ A A
A A A
A
A
C $\mathrm{A}$

5. Data Analysis

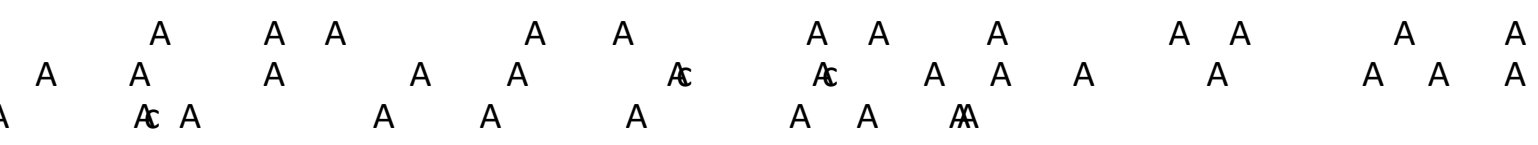

\section{Research Result}

$A$

A $A \quad A$

A A

A $A C \quad A \quad A \quad A \quad A$

$A$ $A \quad A \quad A \quad A$

A A A

$A$ A $A$ A $C$ A A A

A $A$

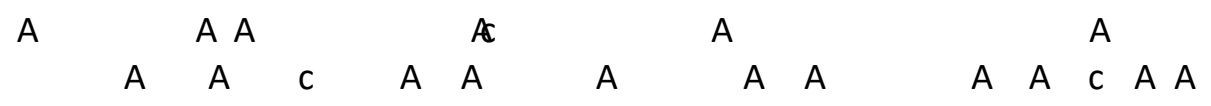

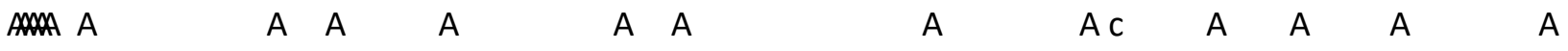
A $A$
A A A A
$\begin{array}{llll}A & A & A & A\end{array}$
A A A
A C A A A
A $A$ A $A$ A
A
A A
A A C A A

A 

A A
A A
A A
A A
A A A
A A
A
A
A A

A A A A A A

A

A $A$

AVew Trends and Issues Proceedings on Humanities and Social Sciences.

Table 1. Score of knowledge about green university of undergraduate students before and after learning. ( $n=40)$

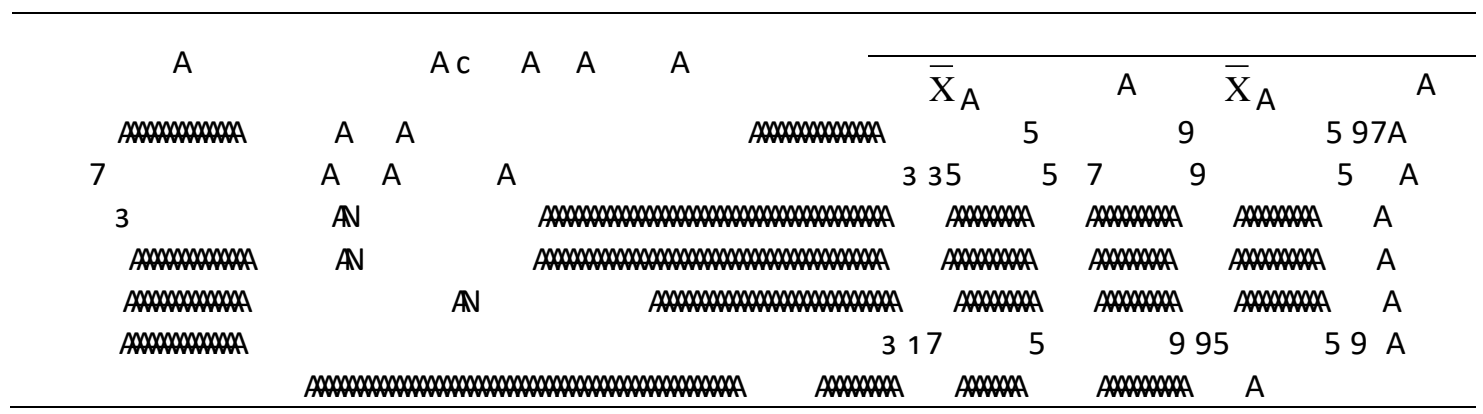

Table 2. Comparison of knowledge about the green university of undergraduate students before and after learning

\begin{tabular}{|c|c|c|c|c|c|c|c|c|c|c|c|c|}
\hline & & & $\overline{\mathrm{X}}_{\mathrm{A}}$ & $\mathrm{A}$ & $A$ & $\bar{X}_{A}$ & & $A$ & $\mathrm{~A}$ & A & & A \\
\hline AOA & & A & A & $A$ & AA & 7 & 13 & & $A$ & 7731 & 3 & $555 \mathrm{~A}$ \\
\hline$A$ & A & $A \quad$ & \multicolumn{3}{|c|}{ - } & $A$ & & & & & & \\
\hline
\end{tabular}

Table 3. Average awareness of green university of undergraduate students before and after learning. $(n=40)$

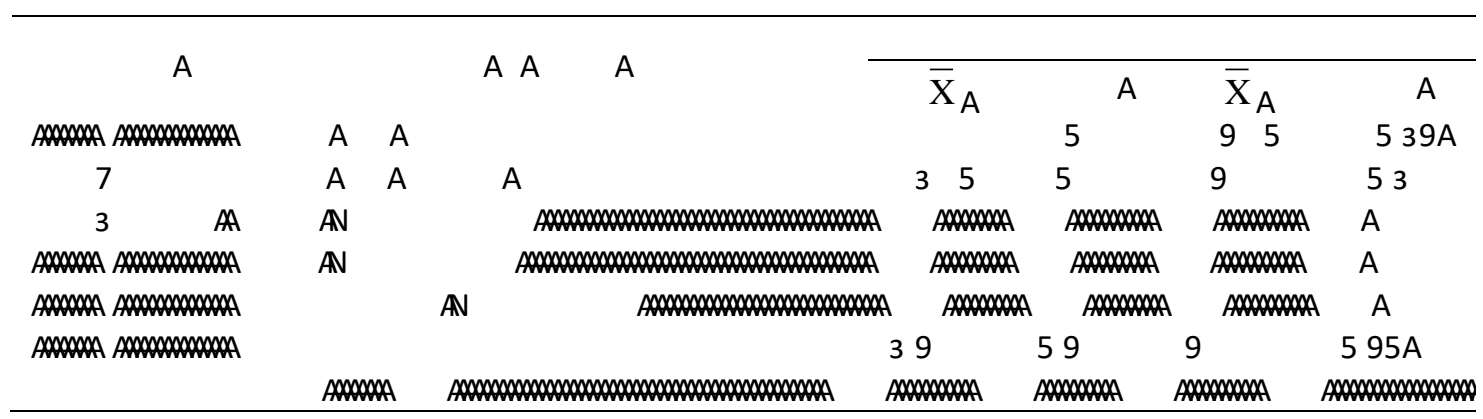

Table 4. Comparison of awareness of green university of undergraduate students, before and after learning

\begin{tabular}{|c|c|c|c|c|c|c|c|c|c|c|c|c|c|}
\hline \multirow[b]{2}{*}{ A } & \multirow{2}{*}{\multicolumn{2}{|c|}{$\bar{X}_{A}$}} & \multirow{2}{*}{$\begin{array}{c}A \\
A N N\end{array}$} & \multirow{2}{*}{$A^{A}$} & \multirow{2}{*}{${ }_{5}^{\bar{X}_{A}}$} & \multirow{2}{*}{51} & \multirow{2}{*}{$\begin{array}{l}A \\
A A\end{array}$} & \multirow{2}{*}{$A^{A}$} & \multirow{2}{*}{1} & \multirow{2}{*}{$\begin{array}{l}\text { A } \\
7\end{array}$} & \multirow{2}{*}{\multicolumn{2}{|c|}{ A }} & A \\
\hline & & & & & & & & & & & & & $A$ \\
\hline & $A$ & $A \mathrm{~A}$ & & & & & & & & & & & \\
\hline
\end{tabular}




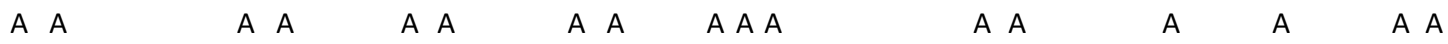

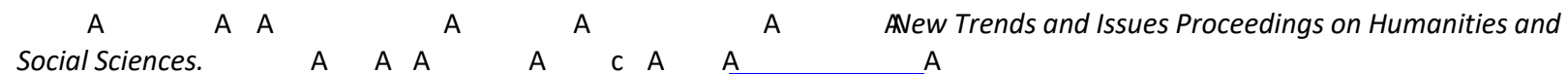

\section{Discussion}

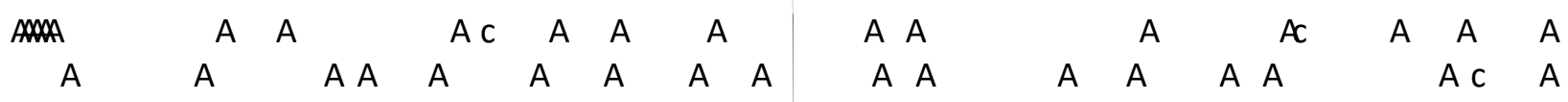
A $A$ A $A$ A A $A$ A A A A A AA A A A A A A $\begin{array}{llllllllllllllll}A & A & A & A & A A & A & A & A A & A & A & A & A & A & A\end{array}$ $\begin{array}{llllllllll}A C & A & C & A & A & A & A & A & A\end{array}$ $\begin{array}{lllllllllllll}A & A & A & A & A & A & A & A & A & A & A & A & A\end{array}$

$A$ A A A A A A A A A A A A A A A A A \begin{tabular}{lll|lll}
$C$ & $C A$ & $A A$ & $A$ & $A$
\end{tabular} AA A A A A A A A A $\quad$ A $A$ A A A $A$ A $A$ A $\begin{array}{llllll}A & & & & & \\ & A & A & A & A & A\end{array}$

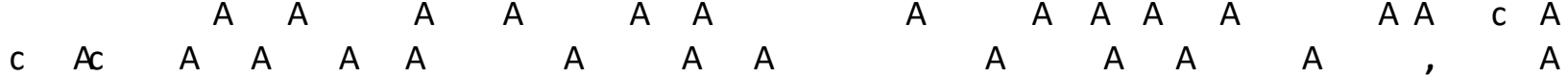
$\begin{array}{lllllllllllll} & A & A & A & A & A & A & A & A & A & A & A\end{array}$

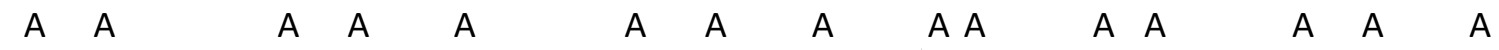
$\begin{array}{lllllllllllllllll}A & A & A & A & A & A & A & A & A & A & A & A & A & A\end{array}$ $\begin{array}{lllllllllll}A & A & A & A & A & A & A & A & A & A & A\end{array}$

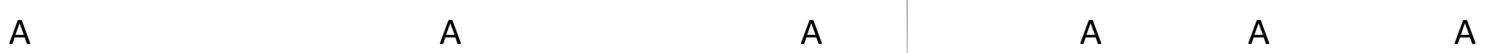
$\begin{array}{lllllllllll}A & A & A & A & A & A & A & A & A & A & A\end{array}$

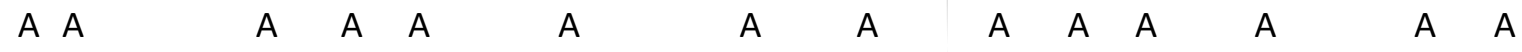
$\begin{array}{lllllllllllll}A & A & A & A & A & A & A & A & A A & A & A & A & A\end{array}$ $\begin{array}{llllll}A & A & A & A & A & A\end{array}$

$\begin{array}{ccccccccccc} & A & A & & A & A & A & & A & & \\ A & A & & A & C & A & A & & A \\ & A & A & & A & A & A & A & \end{array}$

$\begin{array}{llll}A & A & A & A\end{array}$

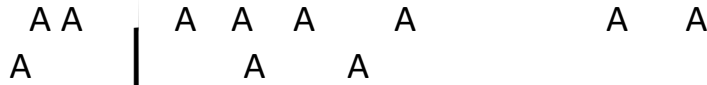

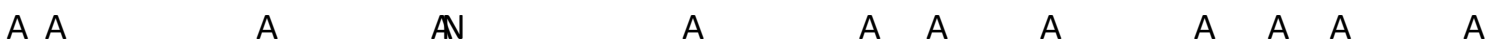

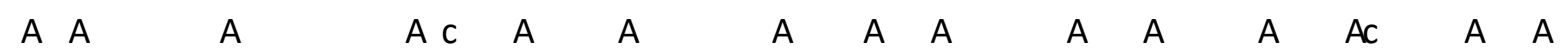
$\begin{array}{lllllllllllll}A & A & A & A & A & A & A & A & A & A & A & A & A\end{array}$

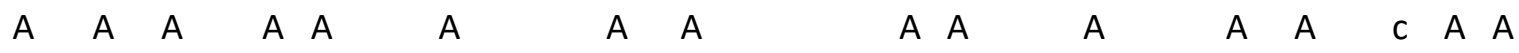
$\begin{array}{llllllllll}A & A & A & A & A & A & A & A & A\end{array}$ $\begin{array}{llllllllllll}A & A & A & A & A & A & A & A & A & A & A & A\end{array}$ $\begin{array}{llllllllllll}A & A C & A & A & A & A & A & A & A & A & A\end{array}$

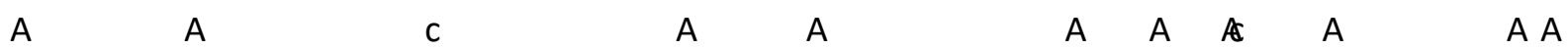
A A A $A$ A $A$ A $\quad A$

$\begin{array}{lllllllllllll}A & A & A & A & A & A & A & A & A & A & A A & A & A\end{array}$

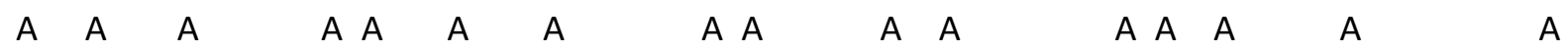

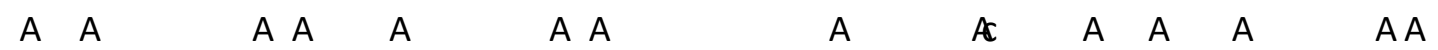
$\begin{array}{lllllllll}A & A & A & A & A & A & A\end{array}$

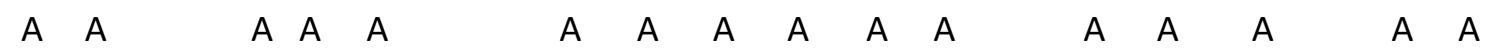
$\begin{array}{lllllllllllll}A A & A & A & A & A & A & A & A & A & A & A & A & A\end{array}$ $\begin{array}{lllllllllllll} & A & A & A & A & A & A & A & A & A\end{array}$

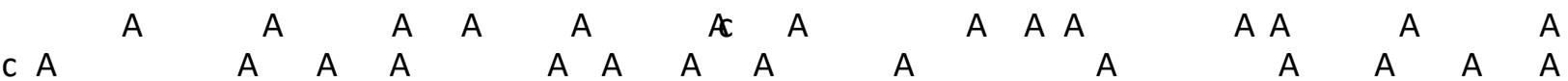
$\begin{array}{lllllllllllllllll}A & A & A & A & A & A A & A & A & A & A & A & A & A & A\end{array}$

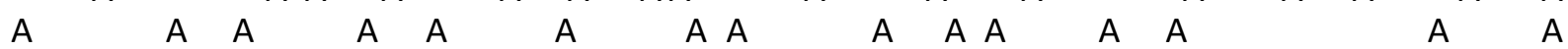




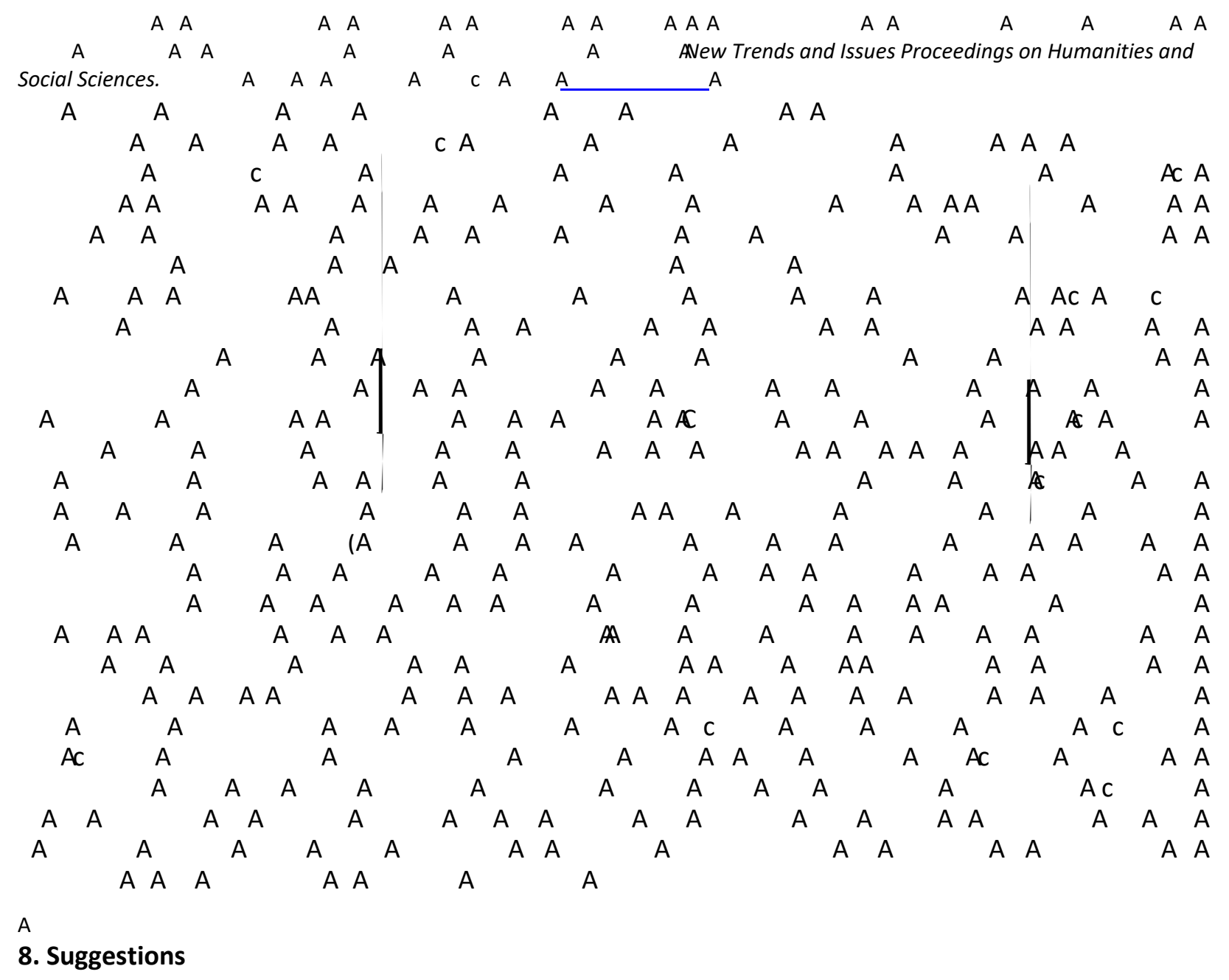

\subsection{Recommendations in the findings}

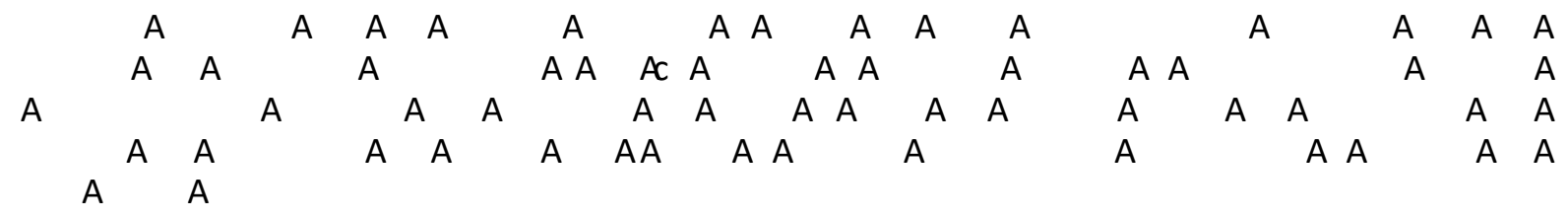

\subsection{The suggestion to do further research}
A $\quad A \quad A \quad A$
A $A$
$A A A \quad A A \quad A \quad A \quad A$
A A A
A A A
A A A
A A A
A A A
$A$
A A A A
A 

A A
A A
A A
A A
A A A
A A
A
A A A

A A A

A A A A $A$ A

$A$

$A$

AVew Trends and Issues Proceedings on Humanities and

Social Sciences.

$\underline{A}$ A

\section{References}

A A A A A A A AOpinions against the baked green university of the development a case study of Faculty of Information Technology, Rajabhat Mahasarakham University. A
A
A AN
A A
A A
A A A C AN
A

C A A

A A A $\quad$ CA A A

A A A

A A

$A$

A A

A

A

A A

C A A A

Attitude structure and function.A

C $A A$

A A A

A A A A

C A

A A A $A$

A $A$

A

Aournal of Education, 9 ( A

A
A A A C A
$A$

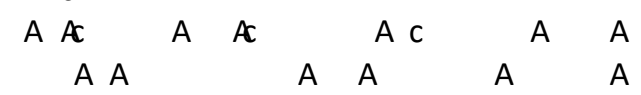

Mahasarakham University, 9 A

$\begin{array}{lrrrrr} & \text { A A } & & \text { Agreen university. A } & \text { A } \\ \text { A A } & \text { A } & \text { A } & \text { A A } & & \text { A }\end{array}$

AVeridian E-Journal Silpakorn University.AB A

A A A A ACU Journal, 18 A

ACA Atatistical methods for research.A A AC

A $A$

$A$

A

A

A

A

A A AMedia development for establishing community awareness and leading to healthy subdistrict by using community participatory development approach: A case study of Mahasawas sub-district, Nakhon Pathom provinceA $\quad$ c $\quad$ A $A$ A A A

A A ABuilding development in Maejo university lead to green university. $\quad$ AN A A A A
A
A A
$\mathbb{A} \quad A$
$A$
A A A
A A A A A A
$A$ A AC A
A C A A
AAEE-T Journal of Environmental Education, 7 A
A A A A

AN A

A A AA 7517

A AA A
$A$ A
AEnvironmental studies.
$A$
A A AEnvironmental education.A
A $\quad A$
A A
A A
A $A$
A A

A

A $A$
A
A A A
AVeridian E-Journal Silpakorn University, 9 ( A
A A
$\mathbb{A}$

A AA A A

A 